\title{
PREVALENCE OF FUNGAL DISEASES IN MEDICINAL PLANTS IN NORTHERN REGION OF BANGLADESH
}

\author{
M.A. Rahman ${ }^{1 *}$, Z.R. Moni $^{2}$, M.A. Hussen ${ }^{2}$ and T.H. Ansari ${ }^{3}$ \\ ${ }^{1}$ Forest Protection Division, Bangladesh Forest Research Institute, Chattogram, Bangladesh. \\ ${ }^{2}$ Bangladesh Agricultural Research Council, Farmgate, Dhaka-1215, Bangladesh. \\ ${ }^{3}$ Principle Scientific Officer, Bangladesh Rice Research Institute, Gazipur-1701, Bangladesh.
}

\begin{abstract}
A season wise survey was conducted on the fungal diseases of some important cultivated medicinal plants in two consecutive years 2014 and 2015 in the northern region of Bangladesh. Eleven fungal diseases namely root rot (Fusarium solani) and leaf blight (Alternaria alternata) of Ashwagandha, leaf spot (Curvularia lunata) and collar rot (Fusarium oxysporium) of Alovera, powdery mildew (Erysiphe sp.) and root rot (Fusarium oxysporium) of Tulsi, collar rot (Fusarium oxysporium) and leaf spot (Collettotrichum gloeosporioides) of Kalmegh, tuberous rot (Fusarium solani) and stem rot (Fusarium oxysporium) of Satamuli, and root rot (Fusarium solani) of Basak were recorded. Most of the diseases were found in the rainy season's comparative to other seasons. Among all the above-mentioned diseases, the powdery mildew (Erysiphe sp.) of Tulsi was found mainly in winter season. With the spreading of these fungal diseases on medicinal plants could be a set back to the industry associated with the formulation of its medicinal products. Therefore, this work may encourage other researchers to study these diseases further for development of proper management.
\end{abstract}

Keywords: Medicinal plant, Survey, Fungal diseases, Causal organism, Disease incidence.

\section{INTRODUCTION}

The importance of medicinal plants is increasing day by day. In recent years, traditional medicine has made a comeback for a variety of reasons including sideeffects and toxicity of modern synthetic drugs, evolution of multi-drug resistance microorganisms, and the inability of modern medicine to find effective cures for a number of diseases. More than $70 \%$ of the developing world's population now depends on traditional medicinal system, otherwise known as complementary or alternative systems of medicine (Azaizeh et al., 2010). In Bangladesh, about 500

\footnotetext{
*Corresponding author: bappy43@yahoo.com
} 
plant species have been identified as medicinal plant because of their therapeutic properties (Ghani, 1998). A large number of industries (400 herbal factories) have been established in this country for producing Ayurvedic, Unani and Kabiraji medicines and about six thousand metric tons of medicinal plants are required annually by the relevant industries in Bangladesh for producing traditional medicines (Sharmin, 2004). There is worth of 11 million US dollars medicinal plant market in Bangladesh (Bregum, 2004). Considering of its market potential, government and some non-government organization have taken mass program to cultivate medicinal plants in to the northern areas of Bangladesh organizing farmers of low-income group, especially the woman. Major cultivated plant species are Ashwagandha (Withaniasomnifera L.), Basak (Adhatodavasica), Tulsi (Ocimum sanctum L.), Kalmegh (Andographispaniculata), Aloevera (Aloe indica L.) and Satamuli (Asparagus racemosus). These medicinally important plants are facing serious problems of the fungal attack. It's adversely affected the medicinal plant parts and decreases the medicinal value of that part. It may be harmful to the human body while using these infected parts as a medicine (Chavan and Korekar, 2011). But in Northern area of Bangladesh the research in diseases of medicinal plants is very few or none. So, identification of the infected fungi is important. Therefore, the purpose of this study was to survey and identify fungal diseases and pathogens associated with the selected medicinal plants of the northern areas of Bangladesh.

\section{MATERIALS AND METHODS}

\section{Survey Area}

Four districts of Rajshahi and Rangpur division which are mid-western and northern region of Bangladesh were selected for the survey study. Natore, Bogra and Jaoypurhat districts of Rajshahi Division and Gaibandha district of Rangpur Division were designated for survey. A total of seven (7) Thana/ Sub-station were surveyed of four selected districts. The selected region has a subtropical monsoon climate characterized by wide seasonal variations in rainfall, moderately warm temperatures and high humidity. Three seasons are generally recognized: a hot, humid summer from April to June; Rainy monsoon season from June to October and a cool, dry winter from October to March. In general, maximum summer temperatures range $32^{\circ} \mathrm{C}-38^{\circ} \mathrm{C}$. April is the warmest month of the country. January is the coldest month, when the average temperature for most of the country is $10^{\circ} \mathrm{C}$. The northern part is mainly agriculture-based area, which is $23 \%$ of total area of Bangladesh and $24 \%$ of total population live here (Banglapedia, 2012). The mass population of northern Bangladesh is under poverty line, struggling hard for a better living standard. Recently, medicinal plants are commercially cultivated (Ashwaganda, Aloevera, Kalmegh, Tulsi, Basak and Satamuli) in the district of Natore, Bogra, Gaibandha, Joypurhat, Rangpur and Naogoan to fulfill the demand of Pharmaceuticals, Ayurvadic and Kabirazi uses. In order to study the disease incidence (\%) of selected medicinal plant was surveyed in summer, rainy and winter season in selected region of Bangladesh during 2014-2015. 


\section{Sample collection, isolation of fungi and estimation of disease incidence}

Leaf blight, leaf spot, root rot, stem rot, powdery mildew, tuberous rot and collar rot samples were collected during field survey. All the samples with the symptoms of disease were collected in separate plastic bags. The name of plant, places and time of samplings were recorded. Samples were taken into the laboratory (Lab.) and the fungi were isolated by using standard moist blotter method (SBM) and Agar plate methods (APM). The survey was conducted on the basis of random sampling method. The disease incidence (\%) was calculated by following formula.

Disease incidence $(\%)=\frac{\text { Number of plants affected }}{\text { Total number of plants observed }} \times 100$

\section{Identification of fungi}

Fungi were identified on the basis of morphological and microscopic characteristics. Pure cultures of these fungi were prepared and maintained on potato dextrose agar (PDA) slants. Plant pathogenic agents of powdery mildews could not grow on most commonly used nutrient media in lab conditions. So, this fungus was identified as such, according to microscopic structures and classified on several related keys and references (Barnett and Hunter, 1999). Pathogenicity tests were carried out in field conditions and the fungi were re-isolated from these plants and identified. The causal organism, morphological and microscopic characteristics of the pathogen and symptoms of the diseases were examined and recorded during experimentation.

\section{Statistical analysis}

All data were analyzed by Duncan's Multiple Range Test (DMRT) using the computer based program SPSS (SPSS Inc., Chicago, IL, USA).

\section{Results and Discussion}

Several fungal diseases were found from the infected medicinal plants in survey areas. The causal organism, morphological and microscopic characteristics of the pathogen and symptoms of the diseases are summarized in Table 1 and 2. After identification of the pathogens the result indicated that the medicinal plant Withania somnifera was attacked by the leaf blight and root rot disease caused by Alternaria alternata and Fusarium solani, respectively. These diseases were found in summer, winter and rainy season. It was observed that the rainy season is more favorable for both diseases. In rainy season, the highest disease incidence (\%) of root rot and leaf blight was recorded 96.74 and $92.16 \%$ at Laxmipur village, Natore sadar thana, Natore district and Mohadipur village, Polashbari thana, Gaibandha district, respectively. The lowest disease incidence (\%) of root rot and leaf blight were recorded 43.87 and $55.64 \%$ at Tilakpur village, Akkelpurthana, Joypurhat district, and Kagoil village, Gabtoli thana, Bogra district, respectively (Table 3). Alternaria leaf spot of Ashwagandha (W. somnifera), caused by A. alternata and root rot wilt 
caused by Fusarium solani is one of the serious diseases of Ashwagandha in the nursery as well as in the commercial fields at Lucknow and its adjoining areas in North India. These diseases may cause the adverse effect on medicinal value of the Withania plant (Gupta et al., 2004). Root rot disease of W. somnifera plant caused by Fusarium solani was also recorded by Rahman et al. (2014) and reported that this fungus can adversely affected at nursery level. Gupta et al. (2004) reported that root rot disease of Ashwagandha caused by Fusarium soalani is one of the destructive diseases and this fungus can affect the nursery plant. The affected plants in the nurseries showed symptoms of yellowing, dropping and decay at seedling stage leading to $30-50 \%$ mortality.

Table 1. Diseases, causal organism and disease symptoms of the some commercially cultivated medicinal plant in northern region of Bangladesh

\begin{tabular}{|c|c|c|c|c|}
\hline Medicinal plant & Local name & Diseases & $\begin{array}{c}\text { Causal } \\
\text { Organism }\end{array}$ & Symptoms \\
\hline \multirow[b]{2}{*}{$\begin{array}{c}\text { Withenia } \\
\text { somnifera } \mathrm{L} \text {. }\end{array}$} & \multirow[b]{2}{*}{$\begin{array}{l}\text { Ashwaga } \\
\text { ndha }\end{array}$} & Root rot & $\begin{array}{l}\text { Fusarium } \\
\text { solani }\end{array}$ & $\begin{array}{l}\text { The root of infected plant showed } \\
\text { pulpiness with brownish color. } \\
\text { Diseased areas of the plant enlarged } \\
\text { with age and turn brown. } \\
\text { Longitudinal cracks were developed } \\
\text { in older lesion and cortical tissues } \\
\text { were discolored and decayed (Fig. } \\
\text { 1a). }\end{array}$ \\
\hline & & $\begin{array}{l}\text { Leaf } \\
\text { blight }\end{array}$ & $\begin{array}{c}\text { Alternaria } \\
\text { alternata }\end{array}$ & $\begin{array}{l}\text { At the initial stage of infection, } \\
\text { symptoms appeared as small, light } \\
\text { brown spots, gradually becoming } \\
\text { irregular, dark brown, concentrically } \\
\text { zonate with a diffuse margin, } \\
\text { frequently surrounded by light yellow } \\
\text { haloes, conspicuous brownish } \\
\text { concentric rings in the advance stage } \\
\text { of infection (Fig. 1b). }\end{array}$ \\
\hline \multirow[t]{2}{*}{ Aloe indica $\mathrm{L}$. } & \multirow[t]{2}{*}{ Aloevera } & Leaf spot & $\begin{array}{l}\text { Curvularia } \\
\text { lunatus }\end{array}$ & $\begin{array}{l}\text { Small, circular to oval dark brown } \\
\text { necrotic sunken spots appeared on the } \\
\text { leaves. As these spots expanded, } \\
\text { centre of the lesion became reddish } \\
\text { brown to brown color. In the advance } \\
\text { stage of infection, spots appeared on } \\
\text { both the surfaces of leaf; affected area } \\
\text { lost the mucilaginous gel and leads } \\
\text { the death of infected leaves (Fig. 1c). }\end{array}$ \\
\hline & & Collar rot & $\begin{array}{c}\text { Fusarium } \\
\text { oxysporium }\end{array}$ & $\begin{array}{l}\text { Small brown spot appeared near } \\
\text { collar region and spread to leaf. As } \\
\text { the disease progressed, growth was } \\
\text { stunted and the affected plants died } \\
\text { (Fig. 1d). }\end{array}$ \\
\hline
\end{tabular}




\begin{tabular}{|c|c|c|c|c|}
\hline Medicinal plant & Local name & Diseases & $\begin{array}{c}\text { Causal } \\
\text { Organism }\end{array}$ & Symptoms \\
\hline \multirow[b]{2}{*}{$\begin{array}{c}\text { Ocimum sanctum } \\
\mathrm{L} .\end{array}$} & \multirow[b]{2}{*}{ Tulsi } & $\begin{array}{l}\text { Powdery } \\
\text { mildew }\end{array}$ & Erysiphe sp. & $\begin{array}{l}\text { The disease first appeared on young } \\
\text { leaves as slightly raised blister like } \\
\text { areas that soon became covered with } \\
\text { greyish, white and powdery growth of } \\
\text { the pathogen on mature leaves (Fig. } \\
\text { 1e). }\end{array}$ \\
\hline & & Root rot & $\begin{array}{c}\text { Fusarium } \\
\text { oxysporium }\end{array}$ & $\begin{array}{l}\text { The lower taproot and lateral roots of } \\
\text { infected plants was appeared brown } \\
\text { to black in color and showed cortical } \\
\text { decay or vascular discoloration. } \\
\text { Lateral roots also died and } \\
\text { decomposed and secondary roots } \\
\text { were developed above them on the } \\
\text { upper taproot (Fig. 1k). }\end{array}$ \\
\hline \multirow{2}{*}{$\begin{array}{l}\text { Andrographis } \\
\text { paniculata }\end{array}$} & \multirow{2}{*}{ Kalmegh } & Collar rot & $\begin{array}{l}\text { Fusarium } \\
\text { oxysporium }\end{array}$ & $\begin{array}{l}\text { The affected plants showed typical } \\
\text { rotting symptoms at the collar region } \\
\text { on which numerous yellowish brown } \\
\text { colored (Fig. } 1 \mathrm{~h} \text { ). }\end{array}$ \\
\hline & & Leaf spot & $\begin{array}{l}\text { Collettotrichu } \\
\text { m } \\
\text { gloeosporioi } \\
\text { des }\end{array}$ & $\begin{array}{l}\text { Infected plants were brown or black } \\
\text { water-soaked spots on the foliage, } \\
\text { sometimes with a yellow halo, } \\
\text { usually uniform in size (Fig. } 1 \mathrm{~g} \text { ). }\end{array}$ \\
\hline \multirow{2}{*}{$\begin{array}{l}\text { Asparagus } \\
\text { racemosus }\end{array}$} & \multirow[b]{2}{*}{ Satamuli } & $\begin{array}{l}\text { Tuberous } \\
\text { rot }\end{array}$ & $\begin{array}{l}\text { Fusarium } \\
\text { solani }\end{array}$ & $\begin{array}{l}\text { Rotten roots were soft and give out } \\
\text { offensive odour particularly in } \\
\text { infections involving pathogen. Stems } \\
\text { became weak at the base and lodge. } \\
\text { (Fig. 1e). }\end{array}$ \\
\hline & & Stem rot & $\begin{array}{l}\text { Fusarium } \\
\text { oxysporium }\end{array}$ & $\begin{array}{l}\text { Initial symptoms showed as pale } \\
\text { yellow lesions at the stem base. These } \\
\text { lesions were expanded and spread to } \\
\text { cause a root and stem rot. Plants were } \\
\text { eventually turned brown and die (Fig. } \\
\text { 1f). }\end{array}$ \\
\hline Adhatoda vasica & Basak & Root rot & $\begin{array}{l}\text { Fusarium } \\
\text { oxysporium }\end{array}$ & $\begin{array}{l}\text { Symptoms were generally included } \\
\text { dark brown to black, discolored, } \\
\text { decaying or completely rotted roots } \\
\text { (Fig. 1i). }\end{array}$ \\
\hline
\end{tabular}


Table 2. Morphological and cultural characteristics of the pathogen

\begin{tabular}{|c|c|c|c|c|}
\hline $\begin{array}{l}\text { Medicinal } \\
\text { plant }\end{array}$ & $\begin{array}{l}\text { Local } \\
\text { name }\end{array}$ & Disease & $\begin{array}{c}\text { Causal } \\
\text { organism }\end{array}$ & Morphological and cultural characteristics \\
\hline \multirow{2}{*}{$\begin{array}{l}\text { Withenia } \\
\text { somnifera } \\
\quad \text { L. }\end{array}$} & \multirow{2}{*}{$\begin{array}{l}\text { Ashwagan } \\
\text { dha }\end{array}$} & Root rot & $\begin{array}{l}\text { Fusarium } \\
\text { solani }\end{array}$ & $\begin{array}{l}\text { Colony grown on PDA became whitish to brown. } \\
\text { Macroconidia were hyaline, two-to several-celled, } \\
\text { fusiform-to sickle-shaped. Microconidia were } 1 \\
\text { to } 2 \text {-celled, hyaline, pyriform, fusiform to ovoid, } \\
\text { straight or curved (Fig } 2 \text { a \& b). }\end{array}$ \\
\hline & & Leaf blight & $\begin{array}{c}\text { Alternaria } \\
\text { alternata }\end{array}$ & $\begin{array}{l}\text { Mycelium was hyaline that turned to grey- } \\
\text { brownish. The conidiophores measured } 42.26 \mu \mathrm{m} \\
(27.30-112 \mu \mathrm{m}) \text { in length and } 4.29 \mu \mathrm{m}(3.12-8.43 \\
\mu \mathrm{m}) \text { in width (Fig. } 2 \mathrm{c} \& \mathrm{~d}) \text {. }\end{array}$ \\
\hline \multirow[b]{2}{*}{$\begin{array}{c}\text { Aloe indica } \\
\mathrm{L} .\end{array}$} & \multirow[b]{2}{*}{ Aloevera } & Leaf spot & $\begin{array}{l}\text { Curvular } \\
\text { ialunata }\end{array}$ & 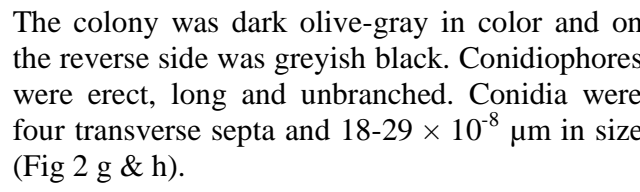 \\
\hline & & Collar rot & $\begin{array}{c}\text { Fusarium } \\
\text { oxysporium }\end{array}$ & $\begin{array}{l}\text { The colonies were white with numerous } \\
\text { microconidia in false heads on short phialides } \\
\text { Microconidia were hyaline, generally single- } \\
\text { celled, oval or cylindrical to slightly curved, and } \\
5.5 \text { to } 17.2 \times 2.6 \text { to } 5.5 \mu \mathrm{m} \text {. Macroconidia were } \\
\text { hyaline, fusiform, slightly to moderately curved } \\
\text { or cylindrical }(2 \mathrm{j} \& \mathrm{k}) \text {. }\end{array}$ \\
\hline \multirow{2}{*}{$\begin{array}{c}\text { Ocimum } \\
\text { sanctum L. }\end{array}$} & \multirow{2}{*}{ Tulsi } & Root rot & $\begin{array}{l}\text { Fusarium } \\
\text { oxysporium }\end{array}$ & $\begin{array}{l}\text { The colony formed with white aerial mycelia. } \\
\text { Microconidia produced on microconidiophores } \\
\text { had elliptical shape and no septate. Macroconidia } \\
\text { were straight to slightly curve in shape with } 3 \\
\text { septa. }\end{array}$ \\
\hline & & $\begin{array}{l}\text { Powdery } \\
\text { mildew }\end{array}$ & Erysiphe sp. & $\begin{array}{l}\text { Conidia were hyaline, ellipsoid to cylindrical, } \\
\text { measuring } 28.1 \text { to } 35.7 \times 12.9 \text { to } 14.4 \mu \mathrm{m} \\
\text { (average } 32.8 \times 13.4 \mu \mathrm{m} \text { ). The conidiophore foot } \\
\text { cell was cylindrical and the appressorium lobed } \\
\text { (Fig. } 2 \text { i). }\end{array}$ \\
\hline \multirow{2}{*}{$\begin{array}{l}\text { Angraphis } \\
\text { peniculata }\end{array}$} & \multirow{2}{*}{ Kalmegh } & Collar rot & $\begin{array}{l}\text { Fusarium } \\
\text { oxysporium }\end{array}$ & $\begin{array}{l}\text { The mycelium was white usually becoming } \\
\text { purple. Microconidia abundant and characteristic } \\
\text { (bean shape). Macroconidia fusiform, slightly } \\
\text { curved. }\end{array}$ \\
\hline & & Leaf spot & $\begin{array}{l}\text { Collettotrichu } \\
\text { m } \\
\text { gloeosporioid } \\
\text { es }\end{array}$ & $\begin{array}{l}\text { Mycellium formed velvety, gray colonies with } \\
\text { slightly wavy margin. The conidiogenous cells } \\
\text { were hyaline, cylindrical, or tapered and } \\
\text { measured up to } 20 \text { by } 3 \text { to } 4 \mu \mathrm{m}(2 \text { e } \& \text { f). }\end{array}$ \\
\hline Asparagus & Satamuli & Tuberous & Fusarium & $\begin{array}{l}\text { The fungus formed white colony with dense aerial } \\
\text { mycelium. Microconidia measuring } 3-16 \times 3-5\end{array}$ \\
\hline
\end{tabular}




\begin{tabular}{|c|c|c|c|c|}
\hline $\begin{array}{l}\text { Medicinal } \\
\text { plant }\end{array}$ & $\begin{array}{l}\text { Local } \\
\text { name }\end{array}$ & Disease & $\begin{array}{c}\text { Causal } \\
\text { organism }\end{array}$ & Morphological and cultural characteristics \\
\hline \multirow[t]{2}{*}{ racemosus } & & rot & solani & $\begin{array}{l}\mu \mathrm{m} \text {. Macroconidia were fusoid with a well- } \\
\text { marked foot cell, and } 5 \text { to } 7 \text { septate measuring } 14- \\
46 \times 3-5 \mu \mathrm{m} \text {. Chlamydospores were globose to } \\
\text { oval, } 6-9 \times 7-10 \mu \mathrm{m} \text {, and terminal or intercalary. }\end{array}$ \\
\hline & & Stem rot & $\begin{array}{l}\text { Fusarium } \\
\text { oxysporium }\end{array}$ & $\begin{array}{l}\text { Of-white floccose (cottony) mycelia observed that } \\
\text { becoming tinged in purple. Microconidia }(5-12 \\
\text { X } 2.3-3.5 \mu \mathrm{m} \text { ) were usually non-septate, } \\
\text { ellipsoidal and were straight. Macroconidia (23- } \\
54 \text { X } 3.0-4.5 \mu \mathrm{m} \text { ) were fusiform in shape. }\end{array}$ \\
\hline $\begin{array}{c}\text { Adhatoda } \\
\text { vasica }\end{array}$ & Basak & Root rot & $\begin{array}{l}\text { Fusarium } \\
\text { oxysporium }\end{array}$ & $\begin{array}{l}\text { The mycelia were white to pink and often with } \\
\text { purple tinge. Microconidia were oval-ellipsoid, } \\
\text { straight to curved, } 5-12 \times 2.2-3.5 \mu \mathrm{m} \text {, and } \\
\text { nonseptate. Macroconidia were three- to five- } \\
\text { septate, fusoid-subulate and pointed at both ends. } \\
\text { Chlamydospores, both smooth and rough walled. }\end{array}$ \\
\hline
\end{tabular}

Aloevera (Aloe indica) plant was affected by the leaf spot disease caused by the Carvularia lunatain in winter, summer and in rainy season. In winter season, the highest disease incidence of leaf spot was recorded $96.75 \%$ at Kantalbaria village, Natore sadar thana, Natore district and the lowest disease incidence was $59.92 \%$ recorded at Harinathpur village, Polashbari thana, Gaibandha district (Table 4). The collar rot disease of Aloevera plant caused by Fusarium oxysporium was also found in all three seasons and the rainy reason is favorable for this disease. In rainy season, the highest disease incidence of collar rot was $89.56 \%$ at Kantalbaria village, Natore sadar thana, Natore district and the lowest disease incidence was recorded $53.28 \%$ at Harinathpur village, Polashbari thana, Gaibandha district (Table 4). The leaf spot disease on Aloe plant first came into limelight as purple spot disease on Aloe arboescens Mill. caused by Fusarium phyllophilum (Kinshi et al., 1999) and later Haematonectria haematococca (anamorph: Fusarium sp.) causing ring spot disease on Aloe barbadensis (Hirooka et al., 2007). Zhai et al. (2013) was first reported of leaf spots in Aloevera caused by Nigrospor aoryzae in China. 
Table 3. Disease incidence (\%) in Ashwagandha at survey areas

\begin{tabular}{|c|c|c|c|c|c|c|c|c|}
\hline \multicolumn{3}{|c|}{ Location } & \multicolumn{6}{|c|}{ Disease incidence $(\%)$} \\
\hline \multirow{3}{*}{ District } & \multirow{3}{*}{ Thana } & \multirow{3}{*}{ Village } & \multicolumn{6}{|c|}{ Seasons } \\
\hline & & & \multicolumn{2}{|c|}{ Summer } & \multicolumn{2}{|c|}{ Winter } & \multicolumn{2}{|c|}{ Rainy } \\
\hline & & & RRA & LBA & RRA & LBA & RRA & LBA \\
\hline \multirow{7}{*}{ Gaibandha } & \multirow{5}{*}{ Sadullapur } & Banagram & $90.12 \mathrm{~b}$ & $75.62 \mathrm{~b}$ & $57.12 \mathrm{a}$ & $58.16 \mathrm{a}$ & $96.26 \mathrm{ab}$ & $88.13 \mathrm{~b}$ \\
\hline & & Dhaperhat & $85.76 \mathrm{~d}$ & $69.95 \mathrm{def}$ & $53.23 \mathrm{c}$ & $54.43 \mathrm{~b}$ & $88.54 \mathrm{~d}$ & 73.65 ef \\
\hline & & Idilpur & $78.97 \mathrm{f}$ & $73.54 \mathrm{c}$ & $46.86 \mathrm{f}$ & $43.86 \mathrm{~d}$ & $87.42 \mathrm{~d}$ & $78.75 \mathrm{c}$ \\
\hline & & Kamarpara & $88.39 \mathrm{c}$ & $60.18 \mathrm{~h}$ & $54.76 \mathrm{bc}$ & $41.76 \mathrm{e}$ & $94.53 \mathrm{bc}$ & $64.75 \mathrm{kl}$ \\
\hline & & Manoharpur & $68.87 \mathrm{jk}$ & $57.86 \mathrm{i}$ & $55.98 \mathrm{ab}$ & $40.32 \mathrm{e}$ & $73.42 \mathrm{ij}$ & 63.531 \\
\hline & \multirow[t]{3}{*}{ Polashbari } & Harinathpur & $75.86 \mathrm{~g}$ & $54.75 \mathrm{j}$ & $54.98 \mathrm{~b}$ & $38.43 \mathrm{~g}$ & $79.66 \mathrm{fg}$ & $68.86 \mathrm{ij}$ \\
\hline & & Mohadipur & $88.28 \mathrm{c}$ & $89.26 \mathrm{a}$ & $46.46 \mathrm{f}$ & $47.24 \mathrm{c}$ & $94.63 \mathrm{bc}$ & $92.16 \mathrm{a}$ \\
\hline \multirow{5}{*}{ Natore } & & Kholabaria & $70.29 \mathrm{ij}$ & $68.16 \mathrm{f}$ & $35.38 \mathrm{j}$ & $36.28 \mathrm{~h}$ & $78.71 \mathrm{~g}$ & $71.28 \mathrm{gh}$ \\
\hline & \multirow{4}{*}{$\begin{array}{l}\text { Natore } \\
\text { sadar }\end{array}$} & Barabaria & $69.54 \mathrm{ijk}$ & $74.21 \mathrm{bc}$ & $32.93 \mathrm{k}$ & $38.12 \mathrm{~g}$ & $76.93 \mathrm{~h}$ & $76.12 \mathrm{~d}$ \\
\hline & & Kantalbaria & $78.19 \mathrm{f}$ & $70.12 \mathrm{de}$ & $40.78 \mathrm{~h}$ & $30.13 \mathrm{kl}$ & $82.93 \mathrm{e}$ & $72.13 \mathrm{fg}$ \\
\hline & & Toltoliapara & $71.18 \mathrm{hi}$ & 68.54 def & $38.23 \mathrm{i}$ & $33.24 \mathrm{i}$ & $80.83 \mathrm{f}$ & 70.42 ghi \\
\hline & & Laxmipur & $92.63 \mathrm{a}$ & $70.38 \mathrm{~d}$ & $50.94 \mathrm{~d}$ & $37.91 \mathrm{gh}$ & $96.74 \mathrm{a}$ & $73.92 \mathrm{e}$ \\
\hline \multirow{4}{*}{ Bogra } & \multirow{2}{*}{$\begin{array}{l}\text { Bogra } \\
\text { sadar }\end{array}$} & Rajapur & $81.18 \mathrm{e}$ & $68.21 \mathrm{f}$ & $42.82 \mathrm{~g}$ & $31.18 \mathrm{j}$ & $84.12 \mathrm{e}$ & $71.16 \mathrm{gh}$ \\
\hline & & Shabgram & $75.87 \mathrm{~g}$ & $54.64 \mathrm{j}$ & $48.97 \mathrm{e}$ & $43.86 \mathrm{~d}$ & $74.65 \mathrm{i}$ & $65.64 \mathrm{k}$ \\
\hline & \multirow{2}{*}{ Gabtoli } & Baliadighi & $68.13 \mathrm{k}$ & $69.54 \mathrm{def}$ & $33.75 \mathrm{k}$ & $34.18 \mathrm{i}$ & $72.23 \mathrm{j}$ & 70.63 ghi \\
\hline & & Kagoil & $72.32 \mathrm{~h}$ & 43.751 & $38.87 \mathrm{i}$ & $39.32 \mathrm{fg}$ & $74.65 \mathrm{i}$ & 55.64 o \\
\hline \multirow{7}{*}{ Joypurhat } & \multirow{4}{*}{ Panchbibi } & Balighata & $69.22 \mathrm{jk}$ & $65.63 \mathrm{~g}$ & $37.98 \mathrm{i}$ & $25.14 \mathrm{~m}$ & $70.21 \mathrm{k}$ & $69.18 \mathrm{ij}$ \\
\hline & & Mohammadpur & 65.541 & $59.98 \mathrm{~h}$ & $43.54 \mathrm{~g}$ & $32.53 \mathrm{ij}$ & $76.86 \mathrm{~h}$ & $64.97 \mathrm{kl}$ \\
\hline & & Kusumba & $43.75 n$ & $54.87 \mathrm{j}$ & $32.65 \mathrm{k}$ & $39.42 \mathrm{fg}$ & $54.43 \mathrm{~m}$ & $61.16 \mathrm{~m}$ \\
\hline & & Dharangi & $53.42 \mathrm{~m}$ & $64.65 \mathrm{~g}$ & $38.87 \mathrm{i}$ & $55.39 \mathrm{~b}$ & 58.961 & $68.12 \mathrm{j}$ \\
\hline & \multirow{3}{*}{ Akkelpur } & Gopinathpur & $36.85 \mathrm{p}$ & 45.761 & $25.65 \mathrm{~m}$ & $32.75 \mathrm{ij}$ & $46.75 n$ & $57.86 \mathrm{n}$ \\
\hline & & Roykali & $42.89 n$ & $49.86 \mathrm{k}$ & 29.871 & 40.31 ef & $53.36 \mathrm{~m}$ & $69.53 \mathrm{hij}$ \\
\hline & & Tilakpur & 39.97 o & $53.32 \mathrm{j}$ & $17.53 n$ & 28.531 & $43.87 \mathrm{o}$ & $57.64 \mathrm{n}$ \\
\hline
\end{tabular}

RRA=Root rot of Ashwagandha, LBA=Leaf Blight of Ashwagandha,

In a column same latters are not significantly different by DMRT at 5\% lavel. 
Table 4. Disease incidence (\%) in Aloevera and Basak at survey areas

\begin{tabular}{|c|c|c|c|c|c|c|c|c|c|c|c|}
\hline \multicolumn{3}{|c|}{ Location } & \multicolumn{9}{|c|}{ Disease incidence $(\%)$} \\
\hline \multirow{3}{*}{ District } & \multirow{3}{*}{ Thana } & \multirow{3}{*}{ Village } & \multicolumn{9}{|c|}{ Seasons } \\
\hline & & & \multicolumn{3}{|c|}{ Summer } & \multicolumn{3}{|c|}{ Winter } & \multicolumn{3}{|c|}{ Rainy } \\
\hline & & & LSA & CRA & RRB & LSA & CRA & RRB & LSA & CRA & RRB \\
\hline \multirow{7}{*}{ Gaibandha } & \multirow{5}{*}{ Sadullapur } & Banagram & $72.53 \mathrm{~g}$ & $\begin{array}{c}75.45 \\
\mathrm{~cd}\end{array}$ & $78.13 \mathrm{~d}$ & $69.76 \mathrm{~d}$ & $65.52 \mathrm{~b}$ & $50.13 \mathrm{~h}$ & $85.92 \mathrm{f}$ & $84.75 \mathrm{~d}$ & $83.72 \mathrm{fg}$ \\
\hline & & Dhaperhat & $65.48 \mathrm{i}$ & $70.21 \mathrm{f}$ & $81.18 \mathrm{c}$ & $61.64 \mathrm{f}$ & $60.18 \mathrm{c}$ & $49.28 \mathrm{hi}$ & $81.16 \mathrm{~g}$ & $75.96 \mathrm{f}$ & $\begin{array}{c}89.19 \\
\text { de }\end{array}$ \\
\hline & & Idilpur & $61.93 \mathrm{j}$ & $58.18 \mathrm{j}$ & $79.32 \mathrm{~d}$ & $59.84 \mathrm{~g}$ & $47.81 \mathrm{~g}$ & $59.41 \mathrm{c}$ & $78.81 \mathrm{~h}$ & $71.64 \mathrm{~g}$ & $85.42 \mathrm{f}$ \\
\hline & & Kamarpara & $58.73 \mathrm{k}$ & $65.72 \mathrm{~g}$ & $76.18 \mathrm{e}$ & $54.73 \mathrm{i}$ & $55.19 \mathrm{~d}$ & $56.73 \mathrm{~d}$ & $76.84 \mathrm{i}$ & $79.73 \mathrm{e}$ & $83.25 \mathrm{~g}$ \\
\hline & & Manoharpur & $45.13 \mathrm{o}$ & $49.13 \mathrm{~m}$ & $72.57 \mathrm{~g}$ & $42.97 \mathrm{~m}$ & $38.92 \mathrm{k}$ & $51.93 \mathrm{~g}$ & 60.731 & $59.43 \mathrm{j}$ & $78.42 \mathrm{ij}$ \\
\hline & \multirow[t]{3}{*}{ Polashbari } & Harinathpur & 44.88 o & $46.62 n$ & $68.53 \mathrm{hi}$ & 41.24 no & 36.141 & $49.18 \mathrm{hi}$ & 59.921 & $53.28 \mathrm{k}$ & $81.21 \mathrm{~h}$ \\
\hline & & Mohadipur & $78.71 \mathrm{e}$ & $55.18 \mathrm{k}$ & $66.93 \mathrm{i}$ & $70.84 \mathrm{~cd}$ & $44.92 \mathrm{~h}$ & $40.32 \mathrm{k}$ & $90.47 \mathrm{~d}$ & $62.53 \mathrm{i}$ & $76.49 \mathrm{k}$ \\
\hline \multirow{5}{*}{ Natore } & & Kholabaria & $86.73 \mathrm{c}$ & $81.53 \mathrm{a}$ & $89.54 \mathrm{a}$ & $79.17 \mathrm{~b}$ & $40.89 \mathrm{j}$ & 38.271 & $93.86 \mathrm{c}$ & $84.65 \mathrm{~d}$ & $96.15 \mathrm{~b}$ \\
\hline & \multirow{4}{*}{$\begin{array}{l}\text { Natore } \\
\text { sadar }\end{array}$} & Barabaria & $89.54 \mathrm{~b}$ & $76.74 \mathrm{c}$ & $79.53 \mathrm{e}$ & $82.64 \mathrm{a}$ & $50.12 \mathrm{f}$ & $35.48 \mathrm{~m}$ & $\begin{array}{c}94.75 \\
\text { bc }\end{array}$ & $87.32 \mathrm{bc}$ & $98.64 \mathrm{a}$ \\
\hline & & Kantalbaria & $90.53 \mathrm{~b}$ & $74.49 \mathrm{~d}$ & $81.63 \mathrm{c}$ & $79.95 \mathrm{~b}$ & $49.93 \mathrm{f}$ & 30.75 o & $96.75 \mathrm{a}$ & $89.56 \mathrm{a}$ & $89.53 \mathrm{~d}$ \\
\hline & & Toltoliapara & $93.53 \mathrm{a}$ & $79.73 \mathrm{~b}$ & $69.63 \mathrm{~h}$ & $83.73 \mathrm{a}$ & $59.96 \mathrm{c}$ & $25.75 \mathrm{p}$ & $\begin{array}{c}95.96 \\
\text { ab }\end{array}$ & $88.43 \mathrm{ab}$ & $91.53 \mathrm{c}$ \\
\hline & & Laxmipur & $85.42 \mathrm{c}$ & $72.18 \mathrm{e}$ & $72.73 \mathrm{~g}$ & $82.75 \mathrm{a}$ & $61.32 \mathrm{c}$ & $53.64 \mathrm{efg}$ & $90.74 \mathrm{~d}$ & $74.54 \mathrm{f}$ & $85.38 \mathrm{f}$ \\
\hline \multirow{5}{*}{ Bogra } & \multirow{2}{*}{ Bogra sadar } & Rajapur & $52.48 \mathrm{~m}$ & 51.931 & $69.95 \mathrm{~h}$ & 45.641 & $45.96 \mathrm{~h}$ & $32.64 n$ & $74.97 \mathrm{j}$ & $64.19 \mathrm{~h}$ & $76.42 \mathrm{k}$ \\
\hline & & Shabgram & $48.93 n$ & 52.171 & $78.95 \mathrm{~d}$ & $41.98 \mathrm{mn}$ & $40.75 \mathrm{j}$ & $54.75 \mathrm{ea}$ & $69.86 \mathrm{k}$ & $59.53 \mathrm{j}$ & $85.53 \mathrm{f}$ \\
\hline & \multirow{3}{*}{ Gabtoli } & Baliadighi & 45.68 o & $60.68 \mathrm{i}$ & $84.63 \mathrm{~b}$ & 39.85 о & $48.86 \mathrm{fg}$ & $72.86 \mathrm{a}$ & $85.64 \mathrm{f}$ & $78.73 \mathrm{e}$ & $89.53 \mathrm{~d}$ \\
\hline & & Kagoil & $49.65 n$ & $74.64 \mathrm{~d}$ & $74.75 \mathrm{ef}$ & $35.97 \mathrm{p}$ & $71.74 \mathrm{a}$ & $67.76 \mathrm{~b}$ & $88.75 \mathrm{e}$ & $86.37 \mathrm{~cd}$ & $94.64 \mathrm{~b}$ \\
\hline & & Balighata & 54.761 & $73.85 \mathrm{~d}$ & $62.53 \mathrm{j}$ & $47.85 \mathrm{k}$ & $65.53 \mathrm{~b}$ & $53.86 \mathrm{ef}$ & $73.96 \mathrm{j}$ & $79.64 \mathrm{e}$ & $78.36 \mathrm{ij}$ \\
\hline \multirow{6}{*}{ Joypurhat } & \multirow{3}{*}{ Panchbibi } & $\begin{array}{c}\text { Mohammadpu } \\
\mathrm{r}\end{array}$ & $49.68 n$ & $65.85 \mathrm{~g}$ & $58.83 \mathrm{k}$ & 39.74 o & $48.75 \mathrm{fg}$ & $49.75 \mathrm{~h}$ & $68.86 \mathrm{k}$ & $72.16 \mathrm{~g}$ & 69.531 \\
\hline & & Kusumba & $75.89 \mathrm{f}$ & $63.98 \mathrm{~h}$ & $62.37 \mathrm{j}$ & $71.95 \mathrm{c}$ & $53.21 \mathrm{e}$ & $52.76 \mathrm{fg}$ & $78.95 \mathrm{~h}$ & $\begin{array}{c}87.96 \\
\text { abc }\end{array}$ & $85.39 \mathrm{f}$ \\
\hline & & Dharangi & $82.95 \mathrm{~d}$ & $61.14 \mathrm{i}$ & $59.65 \mathrm{k}$ & $65.85 \mathrm{e}$ & $49.75 \mathrm{f}$ & $46.86 \mathrm{j}$ & $88.43 \mathrm{e}$ & $75.64 \mathrm{f}$ & $76.75 \mathrm{jk}$ \\
\hline & \multirow{3}{*}{ Akkelpur } & Gopinathpur & $58.86 \mathrm{k}$ & 52.971 & $69.86 \mathrm{~h}$ & $51.64 \mathrm{j}$ & $39.63 \mathrm{jk}$ & $57.12 \mathrm{~d}$ & $69.84 \mathrm{k}$ & $64.63 \mathrm{~h}$ & $87.65 \mathrm{e}$ \\
\hline & & Roykali & $65.74 \mathrm{i}$ & $48.63 \mathrm{~m}$ & $75.53 \mathrm{ef}$ & $59.96 \mathrm{~g}$ & 35.851 & $53.86 \mathrm{ef}$ & $74.85 \mathrm{j}$ & $59.32 \mathrm{j}$ & $89.74 \mathrm{~d}$ \\
\hline & & Tilakpur & $69.74 \mathrm{~h}$ & $49.73 \mathrm{~m}$ & $73.95 \mathrm{fg}$ & $56.52 \mathrm{~h}$ & $42.63 \mathrm{i}$ & $47.65 \mathrm{ij}$ & $85.42 \mathrm{f}$ & $64.89 \mathrm{~h}$ & $79.96 \mathrm{hi}$ \\
\hline
\end{tabular}

LS=Leaf Spot of Aloevera, CRA= Collar Rot of Aloevera, RRB=Root rot of Basak.

In a column same latters are not significantly different by DMRT at 5\% lavel.

Powdery mildew of Tulsi (Ocimum sanctum) caused by the fungus Erysiphe sp. was found only in winter season. The highest disease incidence was recorded $99.16 \%$ at Mohadipur village, Polashbari thana, Gaibandha district and the lowest disease incidence was recorded 53.53\% at Kusumba village, Panchbibi thana, Jaypurhat district (Table 5). The powdery mildew fungi is a significant diseases on a range of crops, and different species of fungi are involved depending on the plant affected (Jahn et al., 2002, Lebeda et al., 2008 and Kristkova et al., 2009). They are important plant pathogens, which are obligate parasitic on the surface of leaves, stems, fruits, 
and flowers of a wide range of angiosperms (Takamatsu et al., 2008). On vegetable crops, powdery mildew usually appears first as yellow spots on the upper leaf surface of older leaves; these spots develop the characteristic powdery growth and symptoms speared to the undersides of leaves and stems (Flint, 1998). Root rot of Tulsi caused by the fungus Fusarium oxysporium was found in summer, winter and in rainy season. The rainy season is more favorable for the disease. In rainy season, the highest disease incidence was recorded $79.96 \%$ at Kamarpara village, Sadullapur thana, Gaibandha district. The lowest disease incidence was recorded $55.75 \%$ at Dharangi village, Panchbibi thana, Joypurhat district (Table 5). Fusariuma is a soil inhabiting pathogen, attacks a large number of host plants including oilseeds, pulses, vegetables and ornamentals (Mani and Sethi, 1968, Bazalar and Delgadi, 1981, Kumar et al., 1983, Kore and Mane, 1992). Fusarium has been contributing significantly for low yield in plant which causes wilting of leaves, tips, loss of turgidity followed by yellowing and drooping of leaves and underground stem become dry, brown and peeling of epidermis. Roots become soft, watery and browning of vascular bundle (Gangopadhyay, 1984).

Collar rot of Kalmegh (Andrographis paniculata) caused by the fungus Fusarium oxysporium was found in summer, winter and in rainy season. The rainy season is more favorable for this disease. In rainy season, the highest and lowest disease incidence was recorded 64.32 and $34.28 \%$ at Tilakpur villagae, Akkelpurthana, Joypurhat district and Kantalbaria village, Natore sadar thana, Natore district, respectively (Table 5). Leaf spot of Kalmegh caused by the fungus Colletotrichum gloeosporioides was also found in summer, winter and in rainy season. The rainy season is more favorable for this disease. In rainy season, the highest disease incidence was recorded $65.84 \%$ at Manoharpur village, Polashbari thana, Gaibandha district and the lowest disease incidence $39.37 \%$ was recorded at Roykali, village, Akkelpurthana, Joypurhat district (Table 5).

Table 5. Disease incidence (\%) in Tulsi and Kalmegh at survey area

\begin{tabular}{|c|c|c|c|c|c|c|c|c|c|c|c|c|c|c|}
\hline \multicolumn{3}{|c|}{ Location } & \multicolumn{12}{|c|}{ Disease incidence (\%) } \\
\hline \multirow{3}{*}{ District } & \multirow{3}{*}{ Thana } & \multirow{3}{*}{ Village } & \multicolumn{12}{|c|}{ Seasons } \\
\hline & & & \multicolumn{4}{|c|}{ Summer } & \multicolumn{4}{|c|}{ Winter } & \multicolumn{4}{|c|}{ Rainy } \\
\hline & & & $\begin{array}{c}\mathrm{PM} \\
\mathrm{T}\end{array}$ & RRT & CRK & LSK & PMT & RRT & CRK & LSK & $\begin{array}{c}\mathrm{PM} \\
\mathrm{T}\end{array}$ & RRT & CRK & LSK \\
\hline \multirow{7}{*}{ Gaibandha } & \multirow{4}{*}{ Sadullapur } & Banagram & - & $56.87 \mathrm{de}$ & $51.74 \mathrm{~b}$ & $35.74 \mathrm{i}$ & $99.64 \mathrm{a}$ & $48.16 \mathrm{~b}$ & $10.13 \mathrm{k}$ & $25.75 \mathrm{~h}$ & - & 67.32 ef & $60.28 \mathrm{bc}$ & $55.86 \mathrm{~d}$ \\
\hline & & Dhaperhat & - & $58.14 \mathrm{~d}$ & $50.61 \mathrm{bc}$ & $32.85 \mathrm{j}$ & $95.14 \mathrm{~b}$ & $42.76 \mathrm{c}$ & $12.29 \mathrm{i}$ & $22.53 \mathrm{i}$ & - & $69.95 \mathrm{~d}$ & 51.43 ef & $46.73 \mathrm{~h}$ \\
\hline & & Idilpur & - & $51.65 \mathrm{hi}$ & $42.21 \mathrm{fg}$ & $30.76 \mathrm{k}$ & $89.28 \mathrm{c}$ & $39.62 \mathrm{~d}$ & $16.72 \mathrm{e}$ & $25.21 \mathrm{~h}$ & - & $62.21 \mathrm{~h}$ & $58.48 \mathrm{c}$ & $48.75 \mathrm{~g}$ \\
\hline & & Kamarpara & - & $68.95 \mathrm{ab}$ & $41.82 \mathrm{~g}$ & $45.85 \mathrm{e}$ & $80.98 \mathrm{f}$ & $58.64 \mathrm{a}$ & $19.36 \mathrm{~d}$ & $28.54 \mathrm{~g}$ & - & $79.96 \mathrm{a}$ & 52.54 de & $54.85 \mathrm{de}$ \\
\hline & \multirow{3}{*}{ Polashbari } & Manoharpur & - & $49.53 \mathrm{jk}$ & $46.84 \mathrm{e}$ & $44.63 \mathrm{ef}$ & $86.29 \mathrm{~d}$ & $44.32 \mathrm{c}$ & $14.73 \mathrm{fg}$ & $23.53 \mathrm{i}$ & - & $59.85 \mathrm{i}$ & $48.68 \mathrm{gh}$ & $65.84 \mathrm{a}$ \\
\hline & & Harinathpur & - & $53.78 \mathrm{~g}$ & $43.72 \mathrm{f}$ & $42.86 \mathrm{f}$ & $82.91 \mathrm{e}$ & $38.43 \mathrm{de}$ & $11.92 \mathrm{ij}$ & $32.85 \mathrm{e}$ & - & $64.63 \mathrm{~g}$ & $48.94 \mathrm{gh}$ & $52.85 \mathrm{f}$ \\
\hline & & Mohadipur & - & $48.69 \mathrm{k}$ & $41.13 \mathrm{gh}$ & $40.63 \mathrm{~g}$ & $99.16 \mathrm{a}$ & $43.74 \mathrm{c}$ & $17.82 \mathrm{de}$ & $26.95 \mathrm{gh}$ & - & $59.95 \mathrm{i}$ & $47.74 \mathrm{~h}$ & $42.17 \mathrm{i}$ \\
\hline \multirow{3}{*}{ Naore } & \multirow{3}{*}{ Natore sadar } & Kholabaria & - & $53.32 \mathrm{gh}$ & $42.64 \mathrm{fg}$ & $56.96 \mathrm{a}$ & $78.26 \mathrm{~g}$ & $48.18 \mathrm{~b}$ & $10.12 \mathrm{k}$ & $32.64 \mathrm{e}$ & - & $55.61 \mathrm{jk}$ & $44.98 \mathrm{i}$ & $64.74 \mathrm{ab}$ \\
\hline & & Barabaria & - & $63.67 \mathrm{c}$ & $30.58 \mathrm{k}$ & $55.84 \mathrm{a}$ & $69.18 \mathrm{i}$ & $24.31 \mathrm{k}$ & $16.26 \mathrm{ef}$ & $43.85 \mathrm{a}$ & - & $74.43 \mathrm{c}$ & $38.16 \mathrm{j}$ & $59.95 \mathrm{c}$ \\
\hline & & Kantalbaria & - & 55.85 ef & $32.12 \mathrm{jk}$ & $48.95 \mathrm{~d}$ & 63.121 & $36.19 \mathrm{f}$ & $10.58 \mathrm{jk}$ & $35.85 \mathrm{~d}$ & - & $68.95 \mathrm{de}$ & $34.28 \mathrm{k}$ & $53.21 \mathrm{ef}$ \\
\hline
\end{tabular}




\begin{tabular}{|c|c|c|c|c|c|c|c|c|c|c|c|c|c|c|}
\hline \multicolumn{3}{|c|}{ Location } & \multicolumn{12}{|c|}{ Disease incidence $(\%)$} \\
\hline \multirow{3}{*}{ District } & \multirow{3}{*}{ Thana } & \multirow{3}{*}{ Village } & \multicolumn{12}{|c|}{ Seasons } \\
\hline & & & \multicolumn{4}{|c|}{ Summer } & \multicolumn{4}{|c|}{ Winter } & \multicolumn{4}{|c|}{ Rainy } \\
\hline & & & \begin{tabular}{|c|}
$\mathrm{PM}$ \\
$\mathrm{T}$
\end{tabular} & RRT & CRK & LSK & PMT & RRT & CRK & LSK & \begin{tabular}{c|}
$\mathrm{PM}$ \\
$\mathrm{T}$
\end{tabular} & RRT & CRK & LSK \\
\hline \multirow{6}{*}{ Bogra } & \multirow{4}{*}{ Bogra sadar } & Toltoliapara & - & $69.64 \mathrm{a}$ & $34.99 \mathrm{i}$ & $53.85 \mathrm{~b}$ & $66.51 \mathrm{j}$ & $58.53 \mathrm{a}$ & $16.23 \mathrm{ef}$ & $40.73 \mathrm{~b}$ & - & $73.21 \mathrm{c}$ & $36.76 \mathrm{j}$ & $59.95 \mathrm{c}$ \\
\hline & & Laxmipur & - & $67.53 \mathrm{~b}$ & 20.281 & $51.32 \mathrm{c}$ & $60.23 \mathrm{~m}$ & $49.79 \mathrm{~b}$ & $13.42 \mathrm{ghi}$ & $43.74 \mathrm{a}$ & - & $77.91 \mathrm{~b}$ & $49.84 \mathrm{fg}$ & $54.73 \mathrm{de}$ \\
\hline & & Rajapur & - & $57.24 \mathrm{de}$ & $30.98 \mathrm{jk}$ & $38.85 \mathrm{~h}$ & $79.18 \mathrm{~g}$ & $42.86 \mathrm{c}$ & $14.33 \mathrm{gh}$ & $32.85 \mathrm{e}$ & - & $65.84 \mathrm{fg}$ & $50.78 \mathrm{f}$ & $48.53 \mathrm{i}$ \\
\hline & & Shabgram & - & $53.78 \mathrm{~g}$ & $41.37 \mathrm{gh}$ & $49.75 \mathrm{~cd}$ & $75.34 \mathrm{~h}$ & $38.75 \mathrm{~d}$ & $13.21 \mathrm{ghi}$ & $38.87 \mathrm{c}$ & & $62.28 \mathrm{~h}$ & $48.69 \mathrm{gh}$ & $53.63 \mathrm{ef}$ \\
\hline & \multirow{2}{*}{ Gabtoli } & Baliadighi & - & $51.19 \mathrm{ij}$ & $40.88 \mathrm{gh}$ & $53.64 \mathrm{~b}$ & $65.54 \mathrm{jk}$ & $39.18 \mathrm{~d}$ & $26.64 \mathrm{~b}$ & $34.25 \mathrm{de}$ & - & $67.95 \mathrm{e}$ & $53.32 \mathrm{~d}$ & $59.53 \mathrm{c}$ \\
\hline & & Kagoil & - & $50.38 \mathrm{ijk}$ & $34.74 \mathrm{i}$ & $55.86 \mathrm{a}$ & $75.88 \mathrm{~h}$ & $33.85 \mathrm{~g}$ & $27.84 \mathrm{~b}$ & $30.86 \mathrm{f}$ & - & $59.32 \mathrm{i}$ & $63.42 \mathrm{a}$ & $64.84 \mathrm{ab}$ \\
\hline \multirow{7}{*}{ Joypurhat } & \multirow{4}{*}{ Panchbibi } & Balighata & - & $49.48 \mathrm{jk}$ & $32.63 \mathrm{j}$ & $43.86 \mathrm{f}$ & $59.53 n$ & $31.64 \mathrm{~h}$ & $19.53 \mathrm{~d}$ & $34.87 \mathrm{~d}$ & - & $56.49 \mathrm{jk}$ & $59.53 \mathrm{c}$ & $53.32 \mathrm{ef}$ \\
\hline & & Mohammadpur & - & $55.85 \mathrm{ef}$ & $39.75 \mathrm{~h}$ & $49.64 \mathrm{~cd}$ & $64.43 \mathrm{kl}$ & $27.85 \mathrm{j}$ & $12.63 \mathrm{hi}$ & $40.64 \mathrm{~b}$ & - & $64.34 \mathrm{~g}$ & $63.76 \mathrm{a}$ & $54.94 \mathrm{de}$ \\
\hline & & Kusumba & - & $53.21 \mathrm{gh}$ & $48.98 \mathrm{~cd}$ & $55.63 \mathrm{a}$ & $53.53 n$ & $43.32 \mathrm{c}$ & $19.53 \mathrm{~d}$ & $38.85 \mathrm{c}$ & - & $68.58 \mathrm{de}$ & $59.53 \mathrm{c}$ & $63.49 \mathrm{~b}$ \\
\hline & & Dharangi & - & $49.87 \mathrm{ijk}$ & $52.52 \mathrm{a}$ & $48.75 \mathrm{~d}$ & $65.95 \mathrm{jk}$ & $32.21 \mathrm{gh}$ & $23.75 \mathrm{c}$ & $34.43 \mathrm{de}$ & - & $55.75 \mathrm{jk}$ & $61.42 \mathrm{~b}$ & $52.85 \mathrm{f}$ \\
\hline & \multirow{3}{*}{ Akkelpur } & Gopinathpur & - & $57.94 \mathrm{~d}$ & $42.31 \mathrm{fg}$ & 44.64 ef & $60.32 \mathrm{~m}$ & $29.63 \mathrm{i}$ & $29.95 \mathrm{a}$ & $39.86 \mathrm{bc}$ & - & $64.26 \mathrm{~g}$ & $60.32 \mathrm{bc}$ & $49.29 \mathrm{~g}$ \\
\hline & & Roykali & - & $54.36 \mathrm{fg}$ & $48.75 \mathrm{~d}$ & $32.86 \mathrm{j}$ & $78.95 \mathrm{~g}$ & 36.85 ef & $31.32 \mathrm{a}$ & $26.43 \mathrm{~h}$ & - & $59.55 \mathrm{i}$ & $59.64 \mathrm{bc}$ & $39.37 \mathrm{j}$ \\
\hline & & Tilakpur & - & $51.28 \mathrm{ij}$ & $53.32 \mathrm{a}$ & $39.86 \mathrm{gh}$ & $84.83 \mathrm{~d}$ & $26.53 \mathrm{j}$ & 16.31 ef & $30.42 \mathrm{f}$ & - & $57.42 \mathrm{j}$ & $64.32 \mathrm{a}$ & $43.53 \mathrm{i}$ \\
\hline
\end{tabular}

PMT=Powdery mildew of Tulsi, RRT=Root rot of Tulsi, CRK= Collar Rot of Kalmegh, LSK= Leaf spot of Kalmegh..

In a column same latters are not significantly different by DMRT at 5\% lavel.

Tuberous rot is a serious disease of Satamuli (Asparagus racemosus). Fusarium solani causes tuberous rot which were found in summer, winter and in rainy season and the highest disease incidence was recorded in rainy season. In rainy season, the highest disease incidence was recorded $91.24 \%$ at Kantalbaria village, Natore sadar thana, Natore district (Table 6). The lowest disease incidence was recorded $47.74 \%$ at Kusumba village, Panchbibi thana, Joypurhat district (Table 6). Stem rot of Satamuli caused by Fusarium oxysporium was also found in summer, winter and in rainy season and the highest disease incidence was recorded in rainy season. In rainy season, the highest disease incidence was $99.18 \%$ at Barabaria village, Natore sadar thana, Natore district and the lowest disease incidence was recorded $54.95 \%$ at Kusumba village, Panchbibi thana, Joypurhat district (Table 6).

Table 6. Disease incidence (\%) of Satamuli at survey areas

\begin{tabular}{|c|c|c|c|c|c|c|c|c|}
\hline \multicolumn{3}{|c|}{ Location } & \multicolumn{6}{|c|}{ Disease incidence $(\%)$} \\
\hline \multirow{3}{*}{ District } & \multirow{3}{*}{ Thana } & \multirow{3}{*}{ Village } & \multicolumn{6}{|c|}{ Seasons } \\
\hline & & & \multicolumn{2}{|c|}{ Summer } & \multicolumn{2}{|c|}{ Winter } & \multicolumn{2}{|c|}{ Rainy } \\
\hline & & & TRS & SRS & TRS & SRS & TRS & SRS \\
\hline \multirow{5}{*}{ Gaibandha } & \multirow{4}{*}{ Sadullapur } & Banagram & $76.26 \mathrm{c}$ & $39.54 \mathrm{jk}$ & $51.26 \mathrm{c}$ & $29.48 \mathrm{j}$ & $88.51 \mathrm{~b}$ & $70.16 \mathrm{j}$ \\
\hline & & Dhaperhat & $67.18 \mathrm{f}$ & 36.241 & $31.28 \mathrm{k} 1$ & $26.58 \mathrm{k}$ & $76.21 \mathrm{e}$ & $68.45 \mathrm{j}$ \\
\hline & & Idilpur & $71.34 \mathrm{~d}$ & $31.43 \mathrm{~m}$ & $49.34 \mathrm{de}$ & 38.49 ef & $80.16 \mathrm{~d}$ & 62.511 \\
\hline & & Kamarpara & $69.22 \mathrm{e}$ & $28.18 \mathrm{n}$ & $38.42 \mathrm{i}$ & $36.73 \mathrm{~g}$ & $78.93 \mathrm{~d}$ & $66.18 \mathrm{k}$ \\
\hline & Polashbari & Manoharpur & $62.38 \mathrm{~h}$ & $24.91 \mathrm{o}$ & $47.83 \mathrm{e}$ & $34.94 \mathrm{~h}$ & $72.16 \mathrm{~g}$ & $90.42 \mathrm{c}$ \\
\hline
\end{tabular}




\begin{tabular}{|c|c|c|c|c|c|c|c|c|}
\hline \multicolumn{3}{|c|}{ Location } & \multicolumn{6}{|c|}{ Disease incidence $(\%)$} \\
\hline \multirow{3}{*}{ District } & \multirow{3}{*}{ Thana } & \multirow{3}{*}{ Village } & \multicolumn{6}{|c|}{ Seasons } \\
\hline & & & \multicolumn{2}{|c|}{ Summer } & \multicolumn{2}{|c|}{ Winter } & \multicolumn{2}{|c|}{ Rainy } \\
\hline & & & TRS & SRS & TRS & SRS & TRS & SRS \\
\hline \multirow{7}{*}{ Natore } & \multirow{7}{*}{ Natore sadar } & Harinathpur & $56.18 \mathrm{j}$ & $26.64 n$ & $40.73 \mathrm{~h}$ & $31.43 \mathrm{i}$ & $69.28 \mathrm{~h} \mathrm{i}$ & $88.73 \mathrm{~d}$ \\
\hline & & Mohadipur & $58.22 \mathrm{i}$ & $45.92 \mathrm{~h}$ & $45.48 \mathrm{f}$ & 24.491 & $65.48 \mathrm{j}$ & $78.89 \mathrm{~g}$ \\
\hline & & Kholabaria & $59.19 \mathrm{i}$ & $40.74 j$ & $42.93 \mathrm{~g}$ & $21.78 \mathrm{~m}$ & $67.87 \mathrm{i}$ & $72.48 \mathrm{i}$ \\
\hline & & Barabaria & $82.81 \mathrm{a}$ & $61.48 \mathrm{a}$ & $43.24 \mathrm{~g}$ & $50.81 \mathrm{a}$ & $89.59 \mathrm{~b}$ & $99.18 \mathrm{a}$ \\
\hline & & Kantalbaria & $79.38 \mathrm{~b}$ & $59.71 \mathrm{~b}$ & $55.22 \mathrm{ab}$ & $49.32 \mathrm{ab}$ & $91.24 \mathrm{a}$ & $96.24 \mathrm{~b}$ \\
\hline & & Toltoliapara & $79.63 \mathrm{c}$ & $51.13 \mathrm{de}$ & $56.38 \mathrm{~b}$ & $47.18 \mathrm{c}$ & $85.16 \mathrm{c}$ & $94.74 \mathrm{~b}$ \\
\hline & & Laxmipur & $76.58 \mathrm{c}$ & 49.52 ef & $53.81 \mathrm{~b}$ & $41.29 \mathrm{~d}$ & $88.16 \mathrm{~b}$ & $91.12 \mathrm{c}$ \\
\hline \multirow{4}{*}{ Bogra } & \multirow{2}{*}{ Bogra sadar } & Rajapur & $65.43 \mathrm{~g}$ & $53.64 \mathrm{c}$ & $43.32 \mathrm{~g}$ & $37.45 \mathrm{fg}$ & $72.65 \mathrm{~g}$ & $85.43 \mathrm{e}$ \\
\hline & & Shabgram & $46.87 \mathrm{~m}$ & $49.86 \mathrm{e}$ & $39.43 \mathrm{hi}$ & $39.53 \mathrm{e}$ & $74.53 \mathrm{f}$ & $81.16 \mathrm{f}$ \\
\hline & \multirow{2}{*}{ Gabtoli } & Baliadighi & $54.13 \mathrm{k}$ & $46.32 \mathrm{gh}$ & $32.17 \mathrm{k}$ & $32.79 \mathrm{i}$ & $69.75 \mathrm{~h}$ & $75.42 \mathrm{~h}$ \\
\hline & & Kagoil & $59.42 \mathrm{i}$ & $47.86 \mathrm{fg}$ & $42.87 \mathrm{~g}$ & $28.38 \mathrm{j}$ & $64.43 \mathrm{jk}$ & $73.85 \mathrm{hi}$ \\
\hline \multirow{7}{*}{ Joypurhat } & \multirow{4}{*}{ Panchbibi } & Balighata & $47.18 \mathrm{~lm}$ & $52.21 \mathrm{~cd}$ & 29.751 & $47.94 \mathrm{bc}$ & 59.851 & $64.63 \mathrm{k}$ \\
\hline & & Mohammadpur & $65.32 \mathrm{~g}$ & $58.96 \mathrm{~b}$ & $34.64 \mathrm{j}$ & 38.64 ef & $69.74 \mathrm{hi}$ & $69.27 \mathrm{j}$ \\
\hline & & Kusumba & $38.96 n$ & $43.86 \mathrm{i}$ & $42.75 \mathrm{~g}$ & $32.75 \mathrm{i}$ & $47.74 \mathrm{~m}$ & $54.95 \mathrm{~m}$ \\
\hline & & Dharangi & 48.641 & 49.43 ef & $40.86 \mathrm{~h}$ & $28.69 \mathrm{j}$ & 58.751 & $64.84 \mathrm{k}$ \\
\hline & \multirow{3}{*}{ Akkelpur } & Gopinathpur & $59.86 \mathrm{i}$ & $38.53 \mathrm{k}$ & $53.75 \mathrm{~b}$ & $17.53 n$ & $63.63 \mathrm{k}$ & $75.42 \mathrm{~h}$ \\
\hline & & Roykali & $65.38 \mathrm{~g}$ & 35.121 & $55.14 \mathrm{ab}$ & $22.31 \mathrm{~m}$ & $69.53 \mathrm{hi}$ & $79.64 \mathrm{fg}$ \\
\hline & & Tilakpur & $69.53 \mathrm{e}$ & $23.41 \mathrm{o}$ & $49.75 \mathrm{~cd}$ & $12.76 \mathrm{o}$ & $73.64 \mathrm{fg}$ & $86.53 \mathrm{e}$ \\
\hline
\end{tabular}

TRS= tuberous rot of Satamuli, SRS $=$ stem rot of Satamuli

In a column same latters are not significantly different by DMRT at 5\% lavel.

Root rot of Basak (Adhatoda vasica) caused by Fusarium oxysporium were found in summer, winter and rainy season. In rainy season the highest disease incidence 98.64(\%) was recorded at Barabaria village, Natore sadar thana, Natore district and the lowest disease incidence $69.53(\%)$ was recorded at Mohammadpur village, Panchbibi thana, Joypurhat district (Table 4).

From the above mentioned results, it was observed that most of the diseases were found in rainy season while in summer season less diseases occurred, it may happen because of favorable temperature and humid conditions. These affected plant parts may be less effective for the medicinal use and economic value. The result is also agreement with on previous study that conducted by Joshi and Kareppa (2010) they studied on disease severity of Chlorophytum borivillianum. They noticed that there is variation in Disease Severity Index (DSI) with respect to season and also mentioned that in rainy season disease severity index was more as compare to other seasons. Shivanna and Mallikarjunasamy (2009) also investigated that the fungal diseases and their effect on phytochemical constituents of medicinally important Terminalia sp. They observed high disease incidence in rainy season next to winter. 


\section{CONCLUSION}

In this study, eleven fungal diseases of six commercially cultivated medicinal plants were recorded in and around different places of Northern region of Bangladesh. The diseases were found more in rainy season while in summer season fewer diseases occurred. The spreading of these fungal diseases on medicinal plants may causes a setback to the industry associated with the formulation of its medicinal products. So, this work may encourage other researcher to study these diseases further and their proper management. It is necessary to maintain the medicinal plants in gardens or nurseries by using bio-pesticides which can make the medicinal plants free from infectious fungal diseases.

\section{REFERENCES}

Anonymous. (1985). Disease assessment manual for crop variety trials. NIAB. Cambridge. Vegetable Keys.

Azaizeh, H., Saad, B., Cooper, E. and Said, O. (2010). Traditional Arabic and Islamic medicine, a re-emerging health aid, Evid Based Complement Alternat Med, 7(4): 419424.

Banglapedia. (2012). National Encyclopedia of Bangladesh.

Barnett, H.L. and Hunter, B.B. (1999). Illustrated genera of imperfect fungi; APS Press. Minnesota.

Bazalar, C.J. and Delgadi, M.A. (1981). Fusarium species associated with the vascular system of cotton (Gossypiumbarbadense L.) under conditions in Piura. Peru. Fitopathologia, 16(1): 6-15.

Bregum, F. (2004). The present status of medicinal plants in Bangladesh, Iranian Journal of Pharmaceutical Research, 94: 3(2): 34-34.

Chavan, S.P. and Korekar, S.L. (2011). A survey of some medicinal plants for fungal diseases from Osmanabad district of Maharashtra State. Recent Research in Science and Technology, 3(5): 15-16.

Flint, M.L. (1988). Pests of the Garden and Small Farm: A Grower's Guide to Using Less Pesticide. Oakland Univ.Calif. Agric. Nat. Res. Publ. p. 3332.

Gangopadhyay, S. (1984). Advance in vegetable disease: S.K. Dutta, Associated Publishing Co., New Delhi. p. 1-5.

Ghani, A. (1998). Medicinal plants of Bangladesh: Chemical constituents and uses. Asiatic society of Bangladesh, Dhaka-1205.

Gupta, M.C., Misra, H.O., Kalra, A. and Khanuja, S.P.S. (2004). Root-knot and wilt: a new disease of Ashwagandha (Withaniasomnifera) caused by Fusariumsolani. Journal of Medicinal and Aromatic Plant Science, 26: 285-287.

Hirooka, Y., Kobayashi, T., Takeuchi, J., Ono, T., Ono, Y. and Natsuaki, K.T. (2007). Aloe ring spot, a new disease of Aloe caused by Haematonectia haematococca (Berg. \& Broome) Samuels and Nirenberg (anamorph: Fusarium sp.). Journal of General Plant Pathology, 73: 330-335. 
Jahn, M., Munger, H.M. and Creight, J.D.M. (2002). Breeding cucurbit crops for powdery mildew resistance. In: R.R. Bélanger., W.R. Bushnell., A.J. Dik. and T.L.W. Carver (eds.), The Powdery Mildews: A Comprehensive Treatise. APS, Press, St. Paul, USA, p. 239-248.

Joshi, A.B. and Kareppa, B.M. (2010). Effect of temperature on growth of seed borne fungi of oil seeds. Variorum, 1(2):1-4.

Kinshi, K., Furukawa, T. and Aoki, T. (1999). Purple spot of Aloe (Aloe arborescens Mill) caused by Fusarium phyllophylum Nirenberg et O'Donnell (New Disease). Annals of the Phytopathological Society of Japan, 65: 576-587.

Kore, S.S. and Mane, A.V. (1992). Dry root rot of kagzilime seedlings caused by Fusarium solani. Journal of Maharashtra Agricultural University, 17(2): 276-278.

Kristkova, E., Lebeda, A. and Sedlakova, B. (2009).Species spectra, distribution and host range of cucurbit powdery mildews in the Czech Republic, and in some other European and Middle Eastern countries. Phytoparasitica, 37:337-350.

Kumar, R.U., Safeeulla, K.M. and Shetty, H.S. (1983). Seed borne nature and infectivity of $F$. solani (Mart.) Sacc. The causal agent to wilt and fruit rot of brinjal. Indian National Science Academic Journal, 49(2): 134-140.

Lebeda, A., Kř́istková E., Sedláková B., McCreight. J.D. and Coffey, M.D. (2008). New concept for determination and denomination of pathotypes and races of cucurbit powdery mildew. Proceedings of the IXth EUCARPIA meeting on genetics and breeding of Cucurbitaceae Avignon, PitratM Editors, France, p. 125-134.

Mani, A. and Sethi, C.L. (1968). Reduced lamina and a typical symptom induced by Fusarium solani on chickpea. Indian Phytopathology, 38: 542- 543.

Rahman, M.A., Islam, M.R. and Nasreen, S. (2014). Screening of Trichoderma strains as a biological control agent against Fusarium solani causing root rot of Ashwagandha (Withania somnifera [L.] Dunal). Bangladesh Journal of Forest Science, 33(1\&2): 110.

Sharmin, L. (2004). Cultivation prospect of medicinal plants in Bangladesh: experiences from Natore; BRAC, Research and Evaluation Division Dhaka, Bangladesh. Pp 41.

Shivanna, M.B. and Mallikarjunaswamy, G.K. (2009). Fungal diseases and their effect on phytochemical constituents of medicinally important Terminalia sp. in Bhadra wildlife sanctuary, Karnataka, India. Indian Phtopath, 62(1): 37-43.

Takamatsu, S., Hirata, T. and Sato, Y. (2008). Phylogenetic analysis and predicted secondary structures of the rDNA internal transcribed spacers of the powdery mildew fungi (Erysiphaceae). Mycoscience, 39: 441-453.

Zhai, L.F., Liu, J., Zhang, M.X., Hong, N., Wang, G.P. and Wang, L.P. (2013). The first report of leaf spots in Aloe vera caused by Nigrospora oryzae in China. Plant Disease, 97 (9): 1256. 

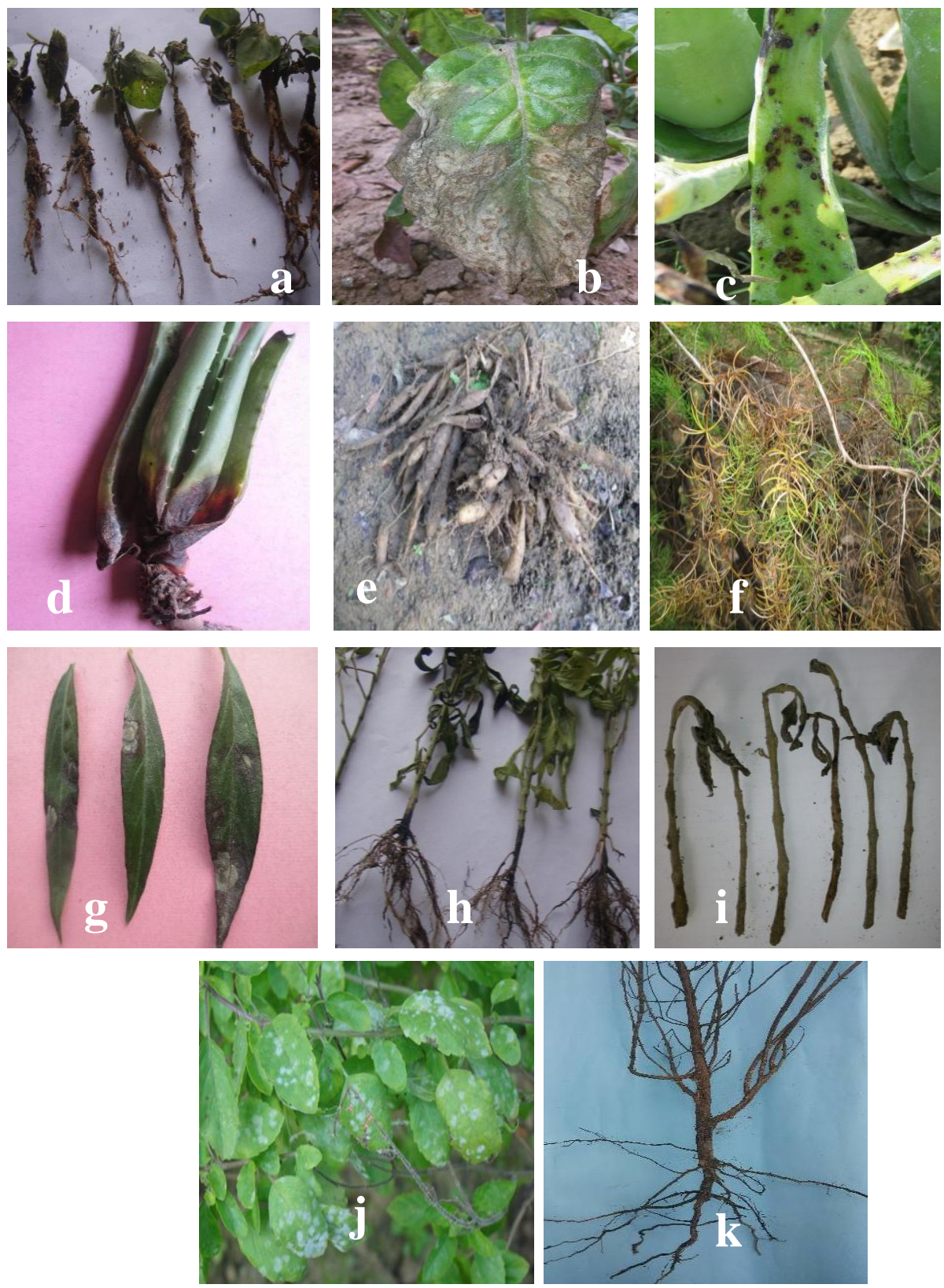

Figure 1: Disease symptoms of six commercially cultivated medicinal plant in northern region of Bangladesh. a \& b: root rot and leaf blight of Ashwagandha; c \& d: leaf spot and collar rot of Aloevera; e \& f: tuberous rot and stem rot of Satamuli; $g$ \& h: leaf spot and collar rot of Kalmegh; i: root rot of Basak; j \& k: Powdery mildew and root rot of Tulsi. 

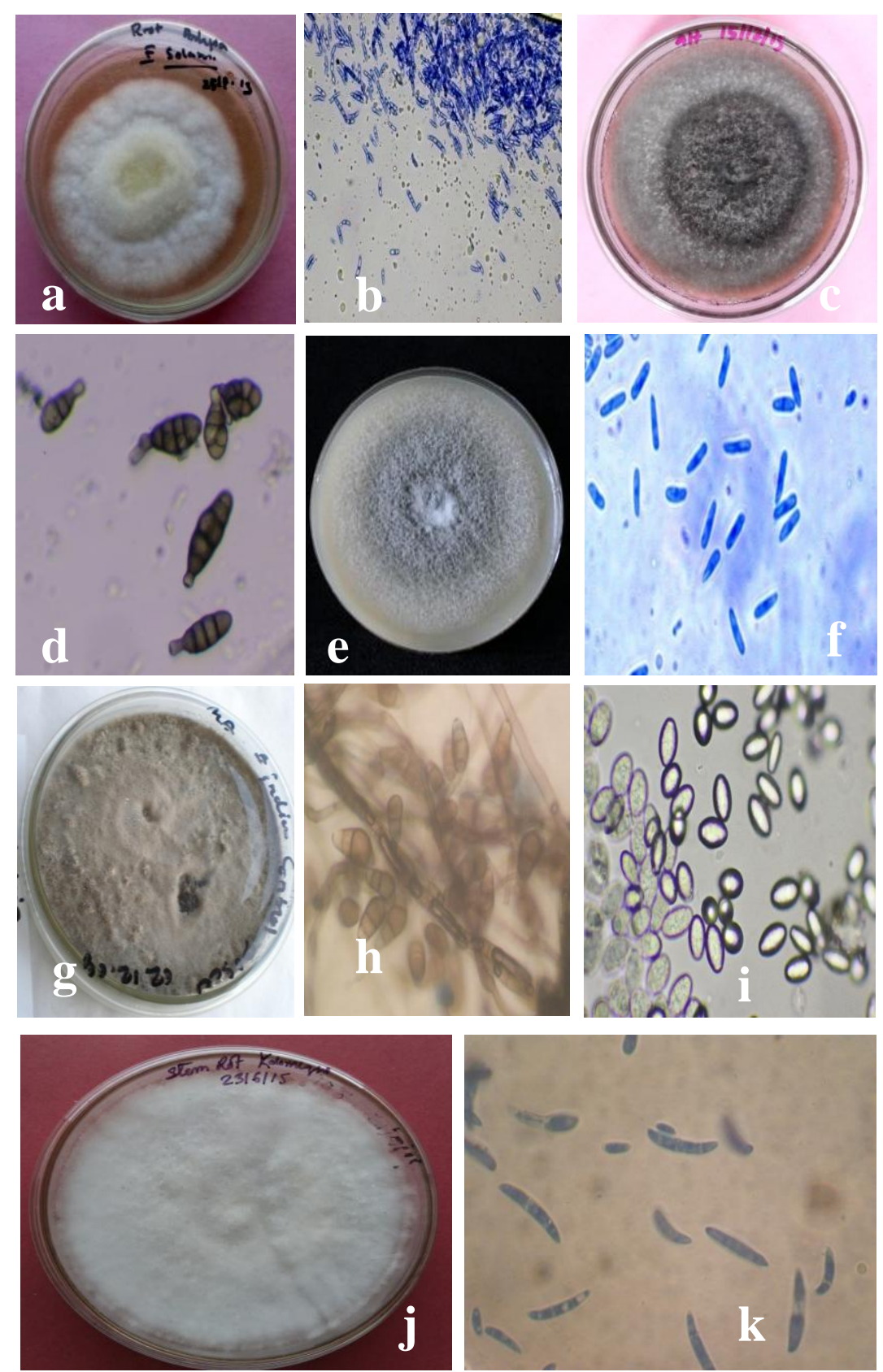

Figure 2: Colony and Conidia of identified fungus. a \& b: F. solani; c \& d: Alternaria alternata; e \& f: Collettotrichum gloeosporioides; $\mathrm{g} \& \mathrm{~h}$ : Curvular ialunata; i : Conidia of Erysiphe sp.; j \& k: Fusarium oxysporium. 